
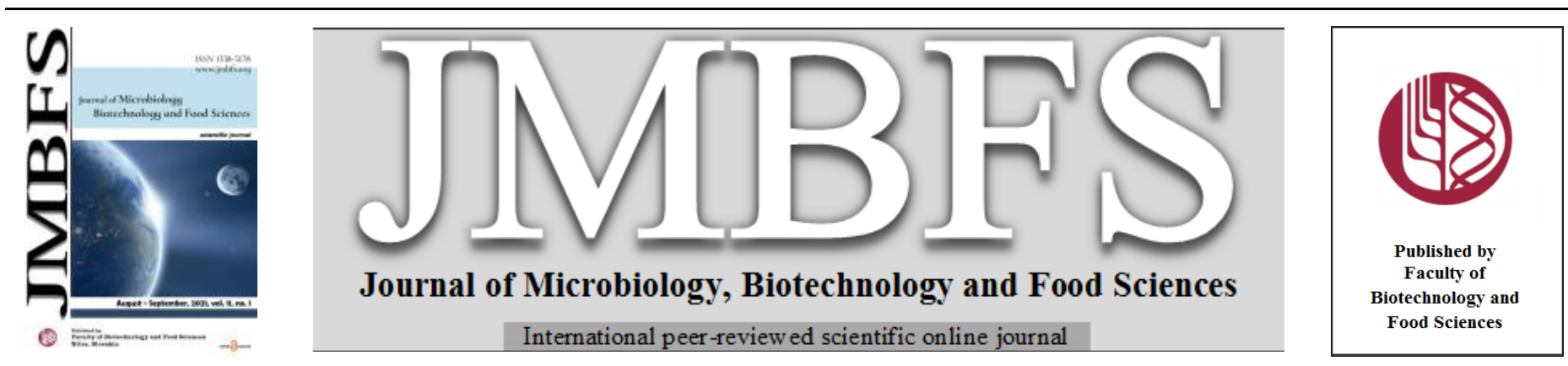

\title{
TAXONOMIC INVESTIGATION OF EUPLANKTONIC DIATOM COMMUNITIES AS INDICATOR OF COPPER IN THE BANK OF THE SUBARNAREKHA RIVER, GHATSHILA, JHARKHAND, INDIA
}

\author{
Gour Gopal Satpati*1, Rahul Bose ${ }^{2}$, Ruma Pal $^{2}$ \\ Address(es): \\ ${ }^{1}$ Department of Botany, Bangabasi Evening College, University of Calcutta, 19 Rajkumar Chakraborty Sarani, Kolkata 700009, West Bengal, India. \\ ${ }^{2}$ Phycology Laboratory, Department of Botany, University of Calcutta, 35 Ballygunge Circular Road, Kolkata 700019, West Bengal, India.
}

*Corresponding author: gour_satpati@yahoo.co.in

https://doi.org/10.15414/jmbfs.2827

\section{ARTICLE INFO}

Received 25. 3. 2020

Revised 20. 3. 2021

Accepted 24. 3. 2021

Published 1. 8. 2021

Regular article

OPEN $\partial_{\text {ACCESS }}$

\begin{abstract}
The aim of this study was to demonstrate and evaluate the diatom communities in the copper infected areas readily associated with the Hindustan Copper Limited (HCL) at the bank of the Subarnarekha River. This study was based on three sampling sites commonly designated as high copper $\left(>100 \mu \mathrm{g} \cdot \mathrm{L}^{-1}\right)$, medium copper $\left(\leq 100 \mu \mathrm{g} . \mathrm{L}^{-1}\right)$ and low copper $\left(\leq 50 \mu \mathrm{g} \cdot \mathrm{L}^{-1}\right)$ contaminated area. Results indicated the detailed taxonomic description of 31 species that are dominant or less dominant over these contaminated area. Among the identified taxa, 10 were recorded as new to the Jharkhand state. Water analysis has suggested the presence of 17 species in the high copper contaminated area adjacent to HCL. Nine species was less dominant in the outlet of HCL that belonged to the medium contaminated and only 5 species were dominant over the low copper contaminated area. Physico-chemical parameters like $\mathrm{pH}$, air and water temperature, salinity, conductivity, light extinction coefficient, turbidity, dissolved inorganic salts, dissolved oxygen and carbondi-oxide, biological oxygen demand and total hardness were also estimated in the copper contaminated sites. Relatively all the species of Cymbella and Navicula were associated with high copper accumulation. Most interestingly, one harmful species Halamphora coffeiformis, which was recorded as most dominant species in high copper exposed area, has shown to be the best copper tolerant and copper indicator species.
\end{abstract}

Keywords: Diatom, copper, indicator, Ghatshila, HCL, taxonomy, tolerant, new record

\section{INTRODUCTION}

Biosorption is a well-known method of phytoremediation, which binds the toxic chemicals or metals and accumulates it in their biological systems especially in cellular structures. The process is widely used to encourage the remediation of heavy metals from the aquatic ecosystems. It also has the potential towards wastewater treatment. Metal toxicity in aquatic ecosystems is commonly triggered by anthropogenic activities including domestic and industrial wastewater, agricultural runoff and dumping of toxic chemicals, e-waste and others (Satpati, 2021). The deposition of toxic elements or trace metals in the water bodies resulting in severe environmental impacts including contamination of surface and ground water and increasing the rate of biomagnification (Sbihi $\boldsymbol{e t}$ al., 2014; Satpati, 2021). Trace metals like copper $(\mathrm{Cu})$ is a well known aquatic pollutant for its adverse affects on phytoplanktons, especially diatoms (Absil, Kroon \& Wolterbeek, 1994). The heavy presence of $\mathrm{Cu}$ in the aquatic food chains may be hazardous to the associated living organisms and to the environment (Nor, 1987). Aquatic living systems may scavenge the trace metals from the water column as well as from the bottom sediments or from both Recently, algae have served as the most potential aquatic living system or bioindicator for accumulating toxic metals (Zeraatkar et al., 2016).

Diatoms belong to the group of Bacillariophyta (Guiry in Guiry \& Guiry, 2021, AlgaeBase), which are frequently used as bioindicators for heavy metals in aquatic bodies. They are unicellular having silicified cell wall. The cell wall consists of two valves held together by a band of girdle. Most of the studies have been done so far on taxonomic documentation. In India, there are many reports on the freshwater diatom flora with detailed taxonomic account (Gandhi, 1959, 1967; Bhakta et al., 2011; Das \& Adhikary, 2012; Dwivedi \& Misra, 2015; Bhakta, Das \& Adhikary, 2016; Bose, Bar \& Pal, 2017). Only few reports on the metal toxicity in diatoms are available. Pandey et al. (2014) have studied the morphological changes of few diatoms exposed to $\mathrm{Cu}$, lead $(\mathrm{Pb})$ and zinc $(\mathrm{Zn})$. Modification of raphe was found more frequent in Fragilaria capucina Gomphonema parvulum, Nitzschia palea, Pinnularia conica and Ulnaria ulna. As diatoms are planktonic, they remain in the open water systems rather in the sediment (Cattaneo et al., 2011). Diatoms are ecologically diverse from centric to pennate form and found in almost all microhabitats in the aquatic ecosystems (Arguelles, 2019). Diatom assemblages can be formed in the open water systems of rivers, lakes and canals (euplanktons), they may be associated with plants (epiphytic), they may be found in the sand (epipsammon), or mud (epipelon) and even in animals (epizooic) (Dixit et al., 1992; Satpati et al., 2017; Arguelles, 2020).

In the present research, the work has been carried out on the taxonomic investigation of some euplanktonic diatoms, which frequently dominate over the $\mathrm{Cu}$ mining area. Hindustan Copper Limited (HCL), situated at the bank of the Subarnarekha River of Ghatshila, is the biggest source of $\mathrm{Cu}$ discharge in the surrounding aquatic habitats. $\mathrm{Cu}$ mining wastes flow directly into the river without any treatment, resulting in significant growth of diatoms and other planktonic organisms. The sampling sites were chosen on the basis of high, medium and low $\mathrm{Cu}$ contamination. Diatom assemblages of these sites were identified and described in detail in relation to abundance. The dominant species from the three different sampling stations were marked on the basis of abundance. Physico-chemical parameters like nitrate, phosphate, silicate, sulphate, calcium, dissolved oxygen (DO), biological oxygen demand (BOD), conductivity, salinity and $\mathrm{pH}$ were also recorded in the present study.

The objective of this study was to determine the diversity of diatom flora as indicator of $\mathrm{Cu}$ in the adjoining water bodies of HCL and Subarnarekha River. In addition, the abundance of the diatom species in terms of low, medium and high $\mathrm{Cu}$ accumulation were also examined. The detailed taxonomic description suggests the proper identification of the euplanktonic diatoms as pollution indicator in aquatic ecosystems. The biochemical assessment of the water has also determined the water quality in the adjacent water bodies of HCL and Ghatshila.

\section{MATERIAL AND METHODS}

\section{Sampling sites}

For the collection of diatom and water samples, four sites were chosen: canal adjacent to HCL, outlets of HCL poured into the Subarnarekha River and the 
river itself commonly designated as Station $1\left(\mathbf{2 2 . 5 9 5 4}^{\circ} \mathbf{N}, \mathbf{8 6 . 4 5 1 9 ^ { \circ }} \mathbf{E}\right)$, Station $2\left(22.5962^{\circ} \mathbf{N}, \mathbf{8 6 . 4 5 2 2}^{\circ} \mathbf{E}\right)$ and Station $3\left(\mathbf{2 1 . 3 3 2 5}^{\circ} \mathbf{N}, \mathbf{8 7 . 2 3 4 1}^{\circ} \mathbf{E}\right)$ respectively. All sampling stations are situated in Ghatshila, Jharkhand (Figure 1).

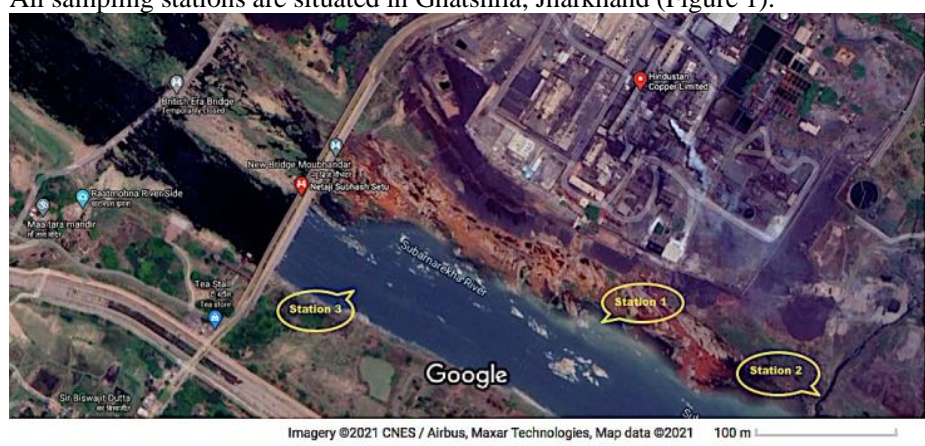

Figure 1 Google satellite image showing three sampling stations (https://www.google.com/maps/search/ghatshila,+hindustan+copper+limited/@2 $2.5911511,86.4454325,4927 \mathrm{~m} /$ data $=! 3 \mathrm{~m} 1 ! 1 \mathrm{e} 3$ )

\section{Diatom collection and preservation}

The copper containing sites associated to Subarnarekha River was investigated in March 2018. The diatom sample was collected through the phytoplankton net of mesh size $25 \mu \mathrm{m}$ (Satpati \& Pal, 2017). After collection, the turbid sample was brought to the laboratory and centrifuged at $10000 \mathrm{rpm}$ for 10 minutes. The pellet thus collected was preserved in $4 \%$ (v/v) formalin for the microscopic study. All the preserved materials were assigned to Calcutta University Herbarium (CUH) voucher specimens.

\section{Water analyses}

Water samples were collected in triplicates at the depth of $0.5 \mathrm{~m}$. The physicochemical parameters were determined with the help of the filtrate obtained from the water samples. All parameters like $\mathrm{pH}$, temperature, electrical conductivity, total hardness, light extinction coefficient, BOD, salinity, nitrate, phosphate, silicate, sulphate and calcium were analyzed using the standard methods of APHA (APHA, 1998). Salinity, pH and temperature were recorded immediately after sampling with ERMA Refractometer (ERMA, Tokyo), Beckman potentiometer zeromatic II and centigrade thermometer respectively. DO content in water sample was estimated in situ following Winkler's Iodometric titration method (Winkler, 1888).

\section{Light Microscopy and Identification}

For light microscopy study, slides were prepared with $20 \%$ glycerin (v/v) and photographs were taken under Carl Zeiss Axioster Plus Microscope by Cannon Power Shot 500D Camera with a coupled micrometer eyepiece (Satpati $\boldsymbol{e t}$ al., 2012, 2013; Satpati $\&$ Pal, 2016). The identification of the species was done using the literature of Hustedt (1930), Hendey (1974), Aboal et al. (2003),

Levkov (2009), Wang et al. (2014), Stepanek \& Kociolek (2018) etc. The classification system was based on the recent up gradation given in AlgaeBase (Guiry in Guiry \& Guiry, 2021)

Cu accumulation study

Table 1 Geospatial and physico-chemical parameters of the $\mathrm{Cu}$ contaminated sites

\begin{tabular}{|c|c|c|c|}
\hline \multirow{2}{*}{$\begin{array}{l}\text { Geospatial and Physico-chemical } \\
\text { parameters }\end{array}$} & \multicolumn{3}{|l|}{ Study sites } \\
\hline & Station 1 (High Cu, $\left(>100 \mu\right.$ g.L L $\left.^{-1}\right)$ & Station 2 (Medium Cu, $\left(\leq 100 \mu \mathrm{g} . \mathrm{L}^{-1}\right)$ & Station $3\left(\right.$ Low $\mathrm{Cu},\left(\leq 50 \mu \mathrm{g} . \mathrm{L}^{-1}\right)$ \\
\hline Coordinates & $22.5954^{\circ} \mathrm{N}, 86.4519^{\circ} \mathrm{E}$ & $22.5962^{\circ} \mathrm{N}, 86.4522^{\circ} \mathrm{E}$ & $21.3325^{\circ} \mathrm{N}, 87.2341^{\circ} \mathrm{E}$ \\
\hline $\mathrm{pH}$ & 6.8 & 7.1 & 7.3 \\
\hline Air temperature $\left({ }^{\circ} \mathrm{C}\right)$ & 32.33 & 32.12 & 32.32 \\
\hline Water temperature $\left({ }^{\circ} \mathrm{C}\right)$ & 28.35 & 29.16 & 30.33 \\
\hline Turbidity (NTU) & 32.22 & 26.33 & 24.21 \\
\hline Electrical conductivity $\left(\mu . S . \mathrm{cm}^{-1}\right)$ & 485.31 & 520.22 & 560.33 \\
\hline Light extinction coefficient (m) & 1.39 & 1.42 & 1.46 \\
\hline Total hardness & 260.33 & 220.22 & 180.35 \\
\hline Salinity (ppt) & 10 & 8 & 6 \\
\hline Dissolved oxygen $\left(\mathrm{mg} \cdot \mathrm{L}^{-1}\right)$ & 2.21 & 2.32 & 3.42 \\
\hline Dissolved $\mathrm{CO}_{2}\left(\mathrm{mg} \cdot \mathrm{L}^{-1}\right)$ & 10.22 & 8.73 & 7.54 \\
\hline Biological oxygen demand (mg. $\left.\mathrm{L}^{-1}\right)$ & 8.83 & 7.43 & 4.45 \\
\hline Nitrate $\left(\mathrm{mg} . \mathrm{L}^{-1}\right)$ & 1.83 & 1.74 & 0.89 \\
\hline Phosphate (mg. $\left.\mathrm{L}^{-1}\right)$ & 0.76 & 0.57 & 0.84 \\
\hline Sulphate $\left(\mathrm{mg} . \mathrm{L}^{-1}\right)$ & 62.32 & 54.48 & 45.22 \\
\hline Silicate $\left(\mathrm{mg} . \mathrm{L}^{-1}\right)$ & 74.44 & 63.22 & 51.51 \\
\hline Calcium $\left(\mathrm{mg} \cdot \mathrm{L}^{-1}\right)$ & 10.42 & 12.55 & 14.75 \\
\hline Copper $\left(\mu \mathrm{g} . \mathrm{L}^{-1}\right)$ & 400 & 94.62 & 47.87 \\
\hline
\end{tabular}

$\mathrm{Cu}$ accumulated in water samples were analyzed using an ICP 2070 Spetrophotometer (Baired, USA) and AAS using a Varian Spectr AA10 apparatus with Graphite Tube Atomizer GTA-95 (Victoria, Australia). The measurement accuracy was checked by the reference of Chmielewska $\&$ Medved (2001)

\section{RESULTS}

\section{Water analyses}

Water analysis report is demonstrated in table 1. During the study, all the sampling stations showed a static air temperature but slightly varied in water temperature. Water temperature was recorded minimum in station 1 with $28.35^{\circ} \mathrm{C}$ whereas highest in Subarnarekha River (station 3) with $30.33^{\circ} \mathrm{C}$. $\mathrm{pH}$ ranges from slightly acidic (below 7.0) to slightly alkaline (above 7.0). The water $\mathrm{pH}$ of the canal adjacent to HCL (station 1) was recorded 6.8. However $\mathrm{pH}$ was recorded highest (7.3) in Subarnarekha River. High turbid condition of the water was noticed in station 1 followed by station 2 and 3 . Electrical conductivity and light extinction coefficient was significantly decreased in the order station 3>station $2>$ station 1. Highest conductivity recorded in Subarnarekha River was $560.33 \mu$ S.cm ${ }^{-1}$. Total hardness varied from 260.33 in station 1 to 180.35 in station 3 . Salinity was recorded highest $(10 \mathrm{ppt})$ in station 1 and lowest $(6 \mathrm{ppt})$ in station 3 . Comparatively DO was highest in station 3 with $3.42 \mathrm{mg} . \mathrm{L}^{-1}$ and lowest in station 1 with $2.21 \mathrm{mg} . \mathrm{L}^{-1}$. However, station 1 recorded highest amount of dissolved $\mathrm{CO}_{2}$ and BOD instead of station 2 and 3. Interestingly nitrate, sulphate and silicate level in the water was high in station 1, which was highly polluted and found adjacent to HCL. Phosphate level in the water of Subarnarekha River was recorded highest $\left(0.84 \mathrm{mg} . \mathrm{L}^{-1}\right)$ and lowest $\left(0.57 \mathrm{mg} . \mathrm{L}^{-1}\right)$ in the outlet of HCL poured directly into the river. Relatively the concentration of the calcium was high in station 3 and low in station 1. Accumulation of $\mathrm{Cu}$ in the water body was recorded highest in station $1\left(400 \mu \mathrm{g} . \mathrm{L}^{-1}\right)$ and lowest in station $3\left(47.87 \mu \mathrm{g} . \mathrm{L}^{-1}\right)$.

\section{Diatom composition}

In the present study, a total number of 31 species were investigated, which belong to 16 families, 10 orders under the class Bacillariophyceae. The diatom composition has suggested the dominance of Cymbella with 6 species followed by 3 species each of Nitzschia and Rhopalodia in the $\mathrm{Cu}$ contaminated area. Two species each from the genus Navicula, Pinnularia, Amphora and Synedra were also documented from the study sites. A large number of species were HCL. From Table 2 it can be obtained that, 17 species are rich in $\mathrm{Cu}$ in Station 1 of which Halamphora coffeiformis was found to be most dominant over the area Similarly this species was absent in station 2 and 3. Interestingly all species of Cymbella and Navicula were reported as high $\mathrm{Cu}$ tolerant species (Table 2). In station 2, nine diatom species were dominant of which both the species of Pinnularia, $P$. acrosphaeria and $P$. viridis showed positive response to $\mathrm{Cu}$ accumulation. Two species each of Rhopalodia and Nitzschia were recommended as $\mathrm{Cu}$ tolerant species in Station 2. However in station 3 only 5 species dominated as Cu tolerant upto $50 \mu \mathrm{g} . \mathrm{L}^{-1}$. Both the species of Synedra, S. ulna and S. ulna var. amphirhynchus were designated as $\mathrm{Cu}$ indicator species in Subarnarekha River. Among the identified diatom species, 10 species viz., Halamphora coffeiformis, Rhopalodia gibberula, Mastogloia smithii var lacustris, Nitzschia nana, Himantidium minus, Synedra ulna var. amphirhynchus, Fragilaria intermedia var. robusta, Grammatophora undulata, Diatoma mesodon and Ctenophora pulchella were recorded as new to the Jharkhand State. documented as $\mathrm{Cu}$ indicator or tolerant in Station 1, closely associated canal of 
Taxonomic description

Phylum: Bacillariophyta

Subphylum: Bacillariophytina

Class: Bacillariophyceae

Subclass: Bacillariophycidae

Order: Naviculales

Suborder: Neidiineae

Family: Diadesmidaceae

Genus: Diadesmis

1. Diadesmis confervacea Kützing [Figures 2a-b]

Aponte, Maidana \& Lange-Bertalot, 2005; Slate \& Stevenson, 2007; Miscoe $e$ al., 2016; Li \& Qi, 2018

Frustules are 2-4 times longer than broad, 10-30 $\mu \mathrm{m}$ long and 5-8 $\mu \mathrm{m}$ broad, rectangular in girdle view, frustules attached side by side to form ribbon shaped colony; striae not distinct under compound microscope.

Voucher no.: CUH/PLANK/DIA- 29/1

Family: Neidiaceae

Genus: Neidium

2. Neidium affine var. amphirhynchus (Ehrenberg) Cleve [Figure 2c]

Hustedt, 1930; Patrick \& Reimer, 1966; Eberle, 2008

Basionym: Navicula amphirhynchus Ehrenberg

Valves 7-8 times longer than broad, 50-70 $\mu \mathrm{m}$ long and 7-10 $\mu \mathrm{m}$ broad,

lanceolate with wide central area with rounded apices.

Voucher no.: CUH/PLANK/DIA- 29/2
Suborder: Naviculineae

Family: Naviculaceae

Genus: Navicula

3. Navicula viridula (Kützing) Ehrenberg [Figure 2d]

Hendey, 1974; Hofmann, Werum \& Lange-Bertalot, 2013; John, 2018

Basionym: Frustulia viridula Kützing

Valves 9-10 times longer than broad, 55-65 $\mu \mathrm{m}$ long and 6-8 $\mu \mathrm{m}$ broad, linear to

lanceolate with capitate ends with narrow axial area and wide central area

Striations are not clear under compound microscope.

Voucher no.: CUH/PLANK/DIA- 29/3

4. Navicula viridula var. rostellata (Kützing) Cleve [Figure 2e]

Patrick \& Reimer, 1966; Hofmann, Werum \& Lange-Bertalot, 2013

Basionym: Navicula rostellata Kützing

Valves narrow, elliptic, lanceolate with short narrowly produced rostrate ends, 4 5 times longer than broad, 38-45 $\mu \mathrm{m}$ long and 9-10 $\mu \mathrm{m}$ broad; axial area narrow and central area big, rounded; striations delicate, radial, approximately 10-12 in $10 \mu \mathrm{m}$ area.

Voucher no.: CUH/PLANK/DIA- 29/4

Family: Amphipleuraceae

Genus: Halamphora

5. Halamphora coffeiformis (C. Agardh) Mereschkowsky [Figure 2f]

Levkov, 2009; Wang et al., 2014; Stepanek \& Kociolek, 2018

Basionym: Frustulia coffeiformis C. Agradh

Table 2 List of $\mathrm{Cu}$ indicating diatoms in three distinct sites (+++ Most dominant, $>70 \%$ of the population; ++ Dominant, 40-70\% of the population; + Less dominant, <40\% of the population; - Absent, No species found)

\begin{tabular}{|c|c|c|c|}
\hline \multirow[b]{2}{*}{ Name of the taxa } & \multicolumn{3}{|c|}{ Cu contaminated area } \\
\hline & $\begin{array}{c}\text { Station 1-High } \\
\text { Cu } \\
\left(>100 \mu \mathrm{g} \mathrm{L}^{-1}\right) \\
\end{array}$ & $\begin{array}{c}\text { Station 2- Medium } \\
\mathrm{Cu} \\
\left(\leq 100 \mu \mathrm{g} \mathrm{L}^{-1}\right)\end{array}$ & $\begin{array}{c}\text { Station 3- Low } \\
\text { Cu } \\
\left(\leq 50 \mu \mathrm{g} \mathrm{L}^{-1}\right)\end{array}$ \\
\hline 1. Diadesmis confervacea Kützing & ++ & + & - \\
\hline 2. Neidium affine var. amphirhynchus (Ehrenberg) Cleve & ++ & + & - \\
\hline 3. Navicula viridula (Kützing) Ehrenberg & ++ & - & - \\
\hline 4. Navicula viridula var. rostellata (Kützing) Cleve & ++ & + & - \\
\hline 5. Halamphora coffeiformis (C. Agardh) Mereschkowsky & +++ & - & - \\
\hline 6. Pinnularia acrosphaeria $\mathrm{W}$. Smith & - & ++ & + \\
\hline 7. Pinnularia viridis (Nitzsch) Ehrenberg & - & ++ & - \\
\hline 8. Rhopalodia gibba (Ehrenberg) O. Müller & ++ & + & + \\
\hline 9. Rhopalodia gibberula (Ehrenberg) O. Müller & - & ++ & - \\
\hline 10. Rhopalodia operculata (C. Agardh) Håkanasson & - & ++ & + \\
\hline 11. Achnanthes exigua Grunow & ++ & + & - \\
\hline 12. Mastogloia smithii var. lacustris Grunow & + & ++ & - \\
\hline 13. Nitzschia obtusa var. scalpelliformis (Grunow) Grunow & + & ++ & - \\
\hline 14. Nitzschia nana Grunow & + & ++ & - \\
\hline 15. Nitzschia acicularis (Kützing) W. Smith & - & + & ++ \\
\hline 16. Amphora elliptica (C. Agardh) Kützing & - & + & ++ \\
\hline 17. Amphora ovum Cleve & ++ & + & - \\
\hline 18. Himantidium minus Kützing & - & + & ++ \\
\hline 19. Synedra ulna (Nitzsch) Ehrenberg & - & + & ++ \\
\hline 20. Synedra ulna var. amphirhynchus (Ehrenberg) Grunow & - & + & ++ \\
\hline 21. Fragilaria intermedia var. robusta G. S. Venkataraman & + & ++ & - \\
\hline 22. Diatoma mesodon (Ehrenberg) Kützing & ++ & + & - \\
\hline 23. Cymbella ehrenbergii Kützing & ++ & + & - \\
\hline 24. Cymbella affinis Kützing & ++ & + & - \\
\hline 25. Cymbella oliffii Cholnoky & ++ & + & - \\
\hline 26. Cymbella cistula (Ehrenberg) O. Kirchner & ++ & - & + \\
\hline 27. Cymbella turgidula Grunow & ++ & - & - \\
\hline 28. Cymbella tumida (Brébisson) Van Heurck & ++ & - & + \\
\hline 29. Gomphonema lanceolatum Ehrenberg, nom. illeg. & ++ & - & + \\
\hline 30. Grammatophora undulata Ehrenberg & ++ & + & - \\
\hline 31. Ctenophora pulchella (Ralfs ex Kützing) D. M. Williams \& Round & + & ++ & - \\
\hline
\end{tabular}



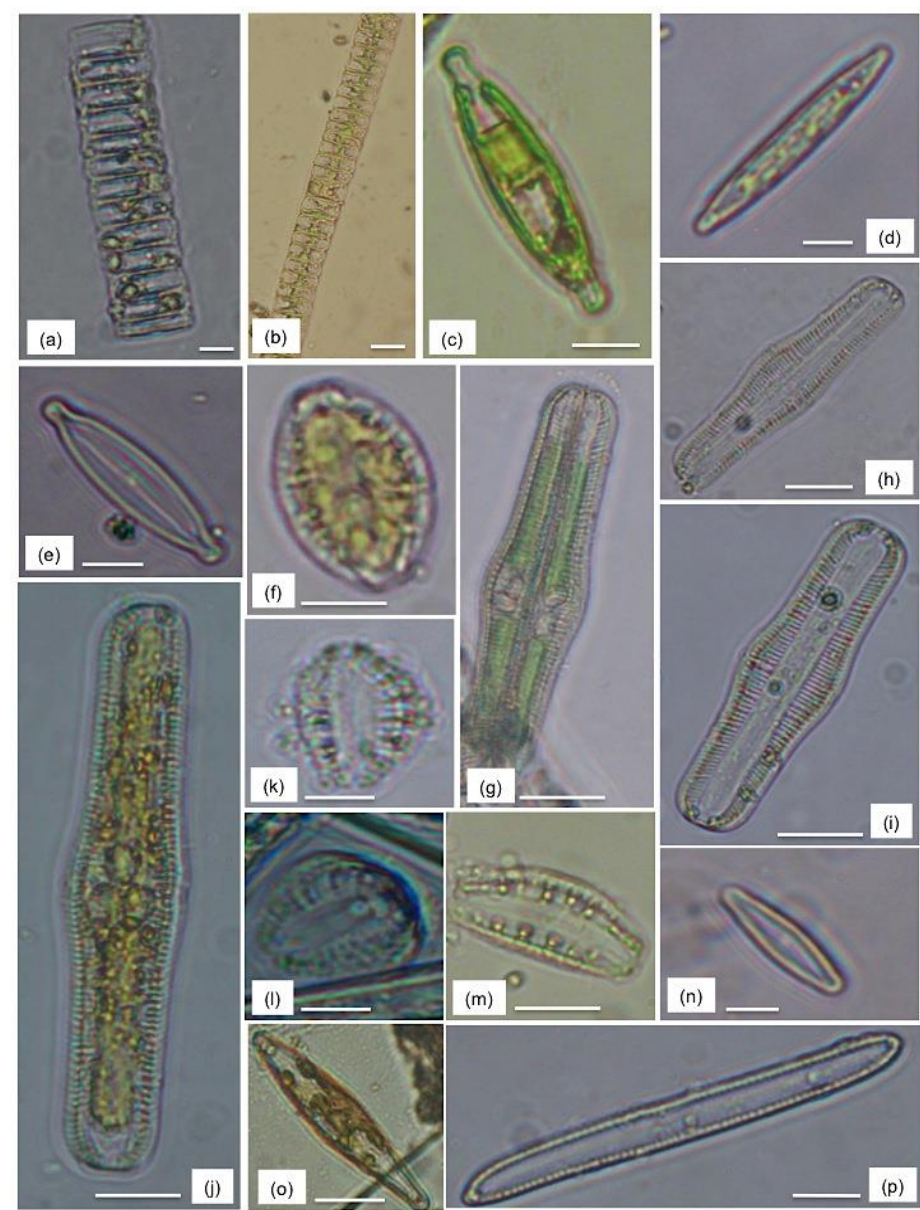

(o)

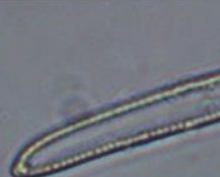

Figure 2 Light micrographs of (a-b) Diadesmis confervacea Kützing. (c) Neidium affine var. amphirhynchus (Ehrenberg) Cleve. (d) Navicula viridula (Kützing) Ehrenberg. (e) Navicula viridula var. rostellata (Kützing) Cleve. (f) Halamphora coffeiformis (C. Agardh) Mereschkowsky. (g) Pinnularia acrosphaeria W. Smith. (h) Pinnularia viridis (Nitzsch) Ehrenberg. (i-j) Rhopalodia gibba (Ehrenberg) O. Müller. (k-1) Rhopalodia gibberula (Ehrenberg) O. Müller. (m) Rhopalodia operculata (C. Agardh) Håkanasson. (n) Achnanthes exigua Grunow. (o) Mastogloia smithii var. lacustris Grunow. (p) Nitzschia obtusa var. scalpelliformis (Grunow) Grunow. (Scale bar a-e, g, i-1, op: $10 \mu \mathrm{m} ; \mathrm{f}, \mathrm{n}: 5 \mu \mathrm{m} ; \mathrm{h}, \mathrm{m}: 20 \mu \mathrm{m})$

Valves semi-lanceolate, dorsal margins, ventral linear, slightly concave with rostrate or capitate apices, 4-6 times longer than broad, 30-50 $\mu \mathrm{m}$ long and 4-12 $\mu \mathrm{m}$ broad; raphe straight, excentric; striae dorsal, coarse, radiate, $8-12$ in $10 \mu \mathrm{m}$ area.

Voucher no.: CUH/PLANK/DIA- 29/5

Suborder: Sellaphorineae

Family: Pinnulariaceae

Genus: Pinnularia

6. Pinnularia acrosphaeria W. Smith [Figure 2g]

Hustedt, 1930; Proschkina-Lavrenko, 1950; Kulikovskiy et al., 2016

Valves linear, gibbous in the middle and at the ends, axial area broad linear and central area punctate, 4.5 to 5.5 times longer than broad, 38-62 $\mu \mathrm{m}$ long and 8-12 $\mu \mathrm{m}$ broad; striations nearly parallel and slightly radial at the ends, striae 9-12 in $10 \mu \mathrm{m}$ area.

Voucher no.: CUH/PLANK/DIA- 29/6

7. Pinnularia viridis (Nitzsch) Ehrenberg [Figure 2h]

Cleve, 1895; Hu \& Wei, 2006; Hofmann, Werum \& Lange-Bertalot, 2013;

Synonym and Basionym: Bacillaria viridis Nitzsch

Valves linear with slightly convex margins and rounded ends, axial area narrow and central area is slightly widened, 5-7 times longer than broad, 90-140 $\mu \mathrm{m}$ long and 18-20 $\mu \mathrm{m}$ broad; raphe complex; striations coarse, slightly radial in the middle and convergent at the ends, striae 7-9 in $10 \mu \mathrm{m}$ area.

Voucher no.: CUH/PLANK/DIA- 30/1

Order: Rhopalodiales

Family: Rhopalodiaceae

Genus: Rhopalodia

8. Rhopalodia gibba (Ehrenberg) O. Müller [Figures 2i-j]

Aboal et al., 2003; Jahn \& Kusber, 2004; Cocquyt, Kusber \& Jahn, 2018

Basionym: Navicula gibba Ehrenberg
Frustules linearly lanceolate with cuneate apices and inflated center, 7-12 times longer than broad, $45-140 \mu \mathrm{m}$ long and 6-12 $\mu \mathrm{m}$ broad; striae distinct $12-18$ in 10 $\mu \mathrm{m}$

Voucher no.: CUH/PLANK/DIA- 30/2

9. Rhopalodia gibberula (Ehrenberg) O. Müller [Figures 2k-I]

Hustedt, 1930; Proschkina-Lavrenko, 1950; John, 2018

Basionym: Eunotia gibberula Ehrenberg

Frustules long, elliptical with rounded ends, 1.5 times longer than broad, sometimes as long as broad, 30-42 $\mu \mathrm{m}$ long and $25-30 \mu \mathrm{m}$ broad, dorsal side highly convex; costae 3-4 in $10 \mu \mathrm{m}$ area.

Voucher no.: CUH/PLANK/DIA- 30/3

10. Rhopalodia operculata (C. Agardh) Håkanasson [Figure 2m]

Ruck et al., 2016; John, 2018

Basionym: Frustulia operculata C. Agardh

Valve linear, solitary, lanceolate or elliptic with wide rounded apices, 3 times longer than broad, 55-60 $\mu \mathrm{m}$ long and 22-24 $\mu \mathrm{m}$ broad; striae transverse, wide apart from each other.

Voucher no.: CUH/PLANK/DIA- 30/4

Order: Mastogloiales

Family: Achnanthaceae

Genus: Achnanthes

11. Achnanthes exigua Grunow [Figure 2n]

Patrick \& Reimer, 1966; Krammer \& Lange-Bertalot, 2004; Hofmann, Werum \& Lange-Bertalot, 2013

Frustules narrow, rectangular in girdle view, forming short chains; valves narrowly lanceolate with clearly convex margins and rostrate apices; valves 5-15 $\mu \mathrm{m}$ long and 3-5 $\mu \mathrm{m}$ broad; striae is not clearly visible under compound microscope

Voucher no.: CUH/PLANK/DIA- 30/5

Family: Mastogloiaceae

Genus: Mastogloia

12. Mastogloia smithii var. lacustris Grunow [Figure 2o]

Proschkina-Lavrenko, 1950; Lee et al., 2014

Valve linear, elliptical and constricted, two narrow ends with central broad area, 4 times longer than broad, 31-32 $\mu \mathrm{m}$ long and 7.5-8.5 $\mu \mathrm{m}$ broad; striations are not clearly visible under compound microscope.

Voucher no.: CUH/PLANK/DIA- 30/6

Order: Bacillariales

Family: Bacillariaceae

Genus: Nitzschia

13. Nitzschia obtusa var. scalpelliformis (Grunow) Grunow [Figure 2p]

Hustedt, 1930; Proschkina-Lavrenko, 1950

Basionym: Nitzschia scalpelliformis Grunow

Valves linear, 10-14 times longer than broad, 80-100 $\mu \mathrm{m}$ long and 6-10 $\mu \mathrm{m}$ broad, apices bend in opposite direction, margins parallel; striae fine 25-30 in 10 $\mu \mathrm{m}$

Voucher no.: CUH/PLANK/DIA- 31/1

14. Nitzschia nana Grunow [Figure 3a]

Hofmann, Werum \& Lange-Bertalot, 2013; Miscoe et al., 2016; John, 2018

Valves linear, 10-15 times longer than broad, 100-200 $\mu \mathrm{m}$ long and 8-12 $\mu \mathrm{m}$ broad, apices sharp niddle like, striae is not clear under compound microscope.

Voucher no.: CUH/PLANK/DIA- 31/2

15. Nitzschia acicularis (Kützing) W. Smith [Figure 3b]

Hustedt, 1930; Aboal et al., 2003; John, 2018

Basionym: Synedra acicularis Kützing

Valves are spindle shaped, slightly silicified, both side of the valves are slightly tapering with sharp narrow apices; valves 15-20 times longer than broad, 35-135 $\mu \mathrm{m}$ long and 2-6 $\mu \mathrm{m}$ broad; striae is not clear under compound microscope.

Voucher no.: CUH/PLANK/DIA- 31/3

Order: Thalassiophysales

Family: Catenulaceae

Genus: Amphora 


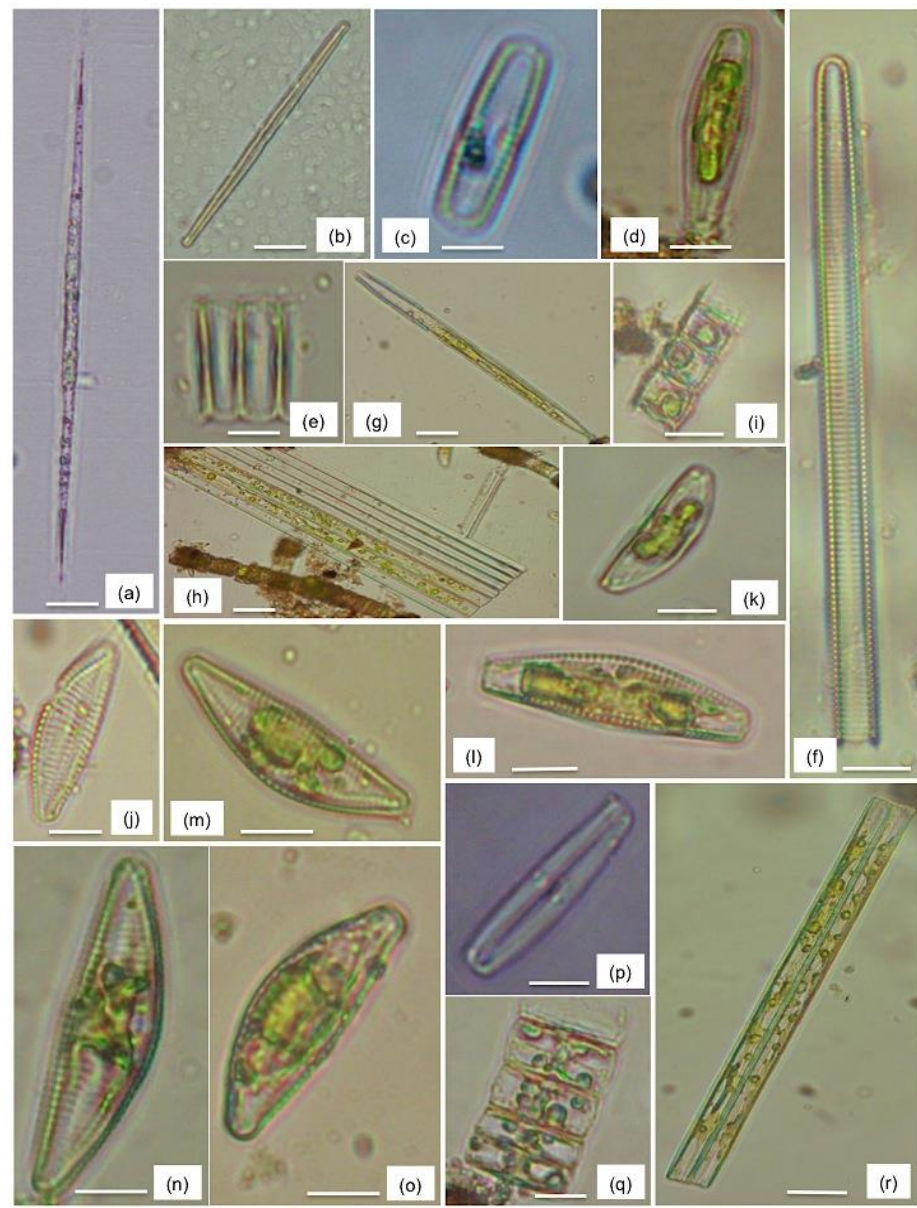

Figure 3 Light micrographs of (a) Nitzschia nana Grunow. (b) Nitzschia acicularis (Kützing) W. Smith. (c) Amphora elliptica (C. Agardh) Kützing. (d) Amphora ovum Cleve. (e) Himantidium minus Kützing. (f) Synedra ulna (Nitzsch) Ehrenberg. (g) Synedra ulna var. amphirhynchus (Ehrenberg) Grunow. (h) Fragilaria intermedia var. robusta G. S. Venkataraman. (i) Diatoma mesodon (Ehrenberg) Kützing. (j) Cymbella ehrenbergii Kützing. (k) Cymbella affinis Kützing. (1) Cymbella oliffii Cholnoky. (m) Cymbella cistula (Ehrenberg) O. Kirchner. (n) Cymbella turgidula Grunow. (o) Cymbella tumida (Brébisson) Van Heurck. (p) Gomphonema lanceolatum Ehrenberg, nom. illeg. (q) Grammatophora undulata Ehrenberg. (r) Ctenophora pulchella (Ralfs ex Kützing) D. M. Williams \& Round. (Scale bar a, q: $20 \mu \mathrm{m}$; b-d, f-1, n, p, r: 10 $\mu \mathrm{m} ; \mathrm{e}, \mathrm{m}, \mathrm{o}: 15 \mu \mathrm{m})$

\section{Amphora elliptica (C. Agardh) Kützing [Figure 3c]}

Henedy, 1974; Aboal et al., 2003

Basionym: Frustulia elliptica C. Agardh

Frustules slightly biconvex, elliptic or lanceolate with faintly attenuated apices, 3 times longer than broad, 26-42 $\mu \mathrm{m}$ long and 8-16 $\mu \mathrm{m}$ broad; striae distinct, transverse at both the sides, 6-8 in $10 \mu \mathrm{m}$ area.

Voucher no.: CUH/PLANK/DIA- 31/4

17. Amphora ovum Cleve [Figure 3d]

Cleve, 1895; Kociolek et al., 2018

Frustules are oval or elliptical with broad rounded apices, 2-3 tomes longer than broad, 20-30 $\mu \mathrm{m}$ long and 9-11 $\mu \mathrm{m}$ broad; striae transverse, generally 5-8 in 10 $\mu \mathrm{m}$

Voucher no.: CUH/PLANK/DIA- 31/5

Class: Bacillariophyta classis incertae sedis

Order: Bacillariophyta ordo incertae sedis

Family: Bacillariophyta familia incertae sedis

Genus: Himantidium

18. Himantidium minus Kützing [Figure 3e]

Van Heurck, 1881; Patrick \& Reimer, 1966

Frustules linear, unilateral and rectangular in girdle view, frustules attached side by side to form ribbon shaped colony, 2 times longer than broad, 30-50 $\mu \mathrm{m}$ long and $15-25 \mu \mathrm{m}$ broad; striations are not clear under compound microscope.

Voucher no.: CUH/PLANK/DIA- 31/6

Subclass: Fragilariophycidae

Order: Fragilariales

Family: Fragilariaceae

Genus: Synedra

19. Synedra ulna (Nitzsch) Ehrenberg [Figure 3f]

Hustedt, 1930; Proschkina-Lavrenko, 1950; Aboal et al., 2003

Basionym: Bacillaria ulna Nitzsch
Frustules are linear, broadened at the ends, 25-30 times longer than broad, 80-160 $\mu \mathrm{m}$ long and 3-7 $\mu \mathrm{m}$ broad; valves linear to lanceolate and gradually tapering towards the ends; central area rounded or rectangular; striae coarse, 10-12 in 10 $\mu \mathrm{m}$.

Voucher no.: CUH/PLANK/DIA- 32/1

20. Synedra ulna var. amphirhynchus (Ehrenberg) Grunow [Figure 3g]

Proschkina-Lavrenko, 1950; Aboal et al., 2003

Basionym: Synedra amphirhynchus Ehrenberg

Valve straight, linear and slender, narrow at the end and suddenly constricted to capitate end, 10-12 times longer than broad; 80-86 $\mu \mathrm{m}$ long and 7-8 $\mu \mathrm{m}$ broad; distinct and parallel striations present in both sides but not prominent in the middle, generally $9-15$ in $10 \mu \mathrm{m}$ area.

Voucher no.: CUH/PLANK/DIA- 32/2

Genus: Fragilaria

21. Fragilaria intermedia var. robusta G. S. Venkataraman [Figure 3h]

Venkataraman, 1939

Frustules are linear, rectangular in girdle view, 14-18 times longer than broad, 70-140 $\mu \mathrm{m}$ long and 5-8 $\mu \mathrm{m}$ broad; valves linear with parallel sides and gradually tapering capitate ends; striae coarse and distinct, $11-12$ in $10 \mu \mathrm{m}$ area.

Voucher no.: CUH/PLANK/DIA- 32/3

Order: Tabellariales

Family: Tabellariaceae

Genus: Diatoma

\section{Diatoma mesodon (Ehrenberg) Kützing [Figure 3i]}

Aboal et al., 2003; Hofmann, Werum \& Lange-Bertalot, 2013; Lange-Bertalot $e t$ al., 2017

Synonym: Odontidium mesodon (Kützing) Kützing

Basionym: Fragilaria mesodon Ehrenberg

Valves are rectangular arranged in chains, $10-20 \mu \mathrm{m}$ long and 7-10 $\mu \mathrm{m}$ broad striae are not clearly visible under compound microscope but usually 20 in $10 \mu \mathrm{m}$ area.

Voucher no.: CUH/PLANK/DIA- 32/4

Order: Cymbellales

Family: Cymbellaceae

Genus: Cymbella

23. Cymbella ehrenbergii Kützing [Figure 3j]

Proschkina-Lavrenko, 1950; Hu \& Wei, 2006

Asymmetrical, biraphid, lanceolate frustules with obtuse end, 2.5 to 3 times longer than broad, 35-36 $\mu \mathrm{m}$ long and 12.5-13 $\mu \mathrm{m}$ broad; transverse raphe located at the middle; central nodule present; distinct transverse striations present and radial towards the center, striae 5-10 in $10 \mu \mathrm{m}$

Voucher no.: CUH/PLANK/DIA- 32/5

\section{Cymbella affinis Kützing [Figure 3k]}

Aboal et al., 2003; Hofmann, Werum \& Lange-Bertalot, 2013

Valves 5 times longer than broad, 20-30 $\mu \mathrm{m}$ long and 4-6 $\mu \mathrm{m}$ broad, strongly dorsiventral with rostrate apices; central stigma present; 8-12 striations in $10 \mu \mathrm{m}$ area.

Voucher no.: CUH/PLANK/DIA- 32/6

\section{Cymbella oliffii Cholnoky [Figure 31]}

Cholnoky, 1956

Frustules are oval to elliptical with large central chloroplast, 3-4 times longer than broad, $40-50 \mu \mathrm{m}$ long and $12-14 \mu \mathrm{m}$ broad; clear transverse striae present, 8 10 in $10 \mu \mathrm{m}$ area.

Voucher no.: CUH/PLANK/DIA- 33/1

26. Cymbella cistula (Ehrenberg) O. Kirchner [Figure 3m]

Hustedt, 1930; Hu \& Wei, 2006

Basionym: Bacillaria cistula Ehrenberg

Valve strongly dorsiventral with rounded apices, 3-5 times longer than broad, 35$80 \mu \mathrm{m}$ long and $10-15 \mu \mathrm{m}$ broad, ventral convex and median inflated; raphe central, proximal, straight; stigma present, 2-3; striae coarse, 8-12 in $10 \mu \mathrm{m}$ area.

Voucher no.: CUH/PLANK/DIA- 33/2

\section{Cymbella turgidula Grunow [Figure 3n]}

Aboal et al., 2003; Hu \& Wei, 2006; John, 2018

Valves dorsiventral, broadly lanceolate, 3 times longer than broad, 30-45 $\mu \mathrm{m}$ long and $10-15 \mu \mathrm{m}$ broad, apices blunt, rostrate to truncate; proximal striae 10-12 and distal striae $12-15$ in $10 \mu \mathrm{m}$ area.

Voucher no.: CUH/PLANK/DIA- 33/3

28. Cymbella tumida (Brébisson) Van Heurck [Figure 3o]

Aboal et al., 2003; Hu \& Wei, 2006; Hofmann, Werum \& Lange-Bertalot, 2013

Valves broadly lanceolate with obtuse ends, 3-5 times longer than broad, 50-70 $\mu \mathrm{m}$ long and 10-20 $\mu \mathrm{m}$ broad; dorsal and ventral margins of the valve bent in opposite direction; striations radial, 8-10 in $10 \mu \mathrm{m}$ area

Voucher no.: CUH/PLANK/DIA- 33/4

Family: Gomphonemataceae

Genus: Gomphonema

29. Gomphonema lanceolatum Ehrenberg, nom. illeg. [Figure 3p] Hustedt, 1930; Eberle, 2008

Valves lanceolate to clavate in shape with distinctly rounded apex and base, 4-4.5 time longer than broad, 50-55 $\mu \mathrm{m}$ long and $12-14 \mu \mathrm{m}$ broad; raphe slightly thick and straight; Striae radial and lineate, 10 in $10 \mu \mathrm{m}$ area. 
Voucher no.: CUH/PLANK/DIA- 33/5

Order: Rhabdonematales

Family: Grammatophoraceae

Genus: Grammatophora

30. Grammatophora undulata Ehrenberg [Figure 3q]

Proschkina-Lavrenko, 1950; Witkowski, Lange-Bertalot \& Metzeltin, 2000; Cheng \& Gao, 2012

Frustules quadrangular with rounded angles, 2 times longer than broad, $40-45 \mu \mathrm{m}$ long and $20-23 \mu \mathrm{m}$ broad; septa slightly undulated; valves linear, oblong with capitulate ends; striations transverse, 6-8 in $10 \mu \mathrm{m}$ area.

Voucher no.: CUH/PLANK/DIA -33/6

Order: Licmophorales

Family: Ulnariaceae

Genus: Ctenophora

31. Ctenophora pulchella (Ralfs ex Kützing) D. M. Williams \& Round [Figure 3r]

Aboal et al., 2003; Hofmann, Werum \& Lange-Bertalot, 2013

Basionym: Exilaria pulchella Ralfs ex Kützing

Frustules form ribbon shaped colony, 40-50 times longer than broad, 150-200 $\mu \mathrm{m}$ long and 5-7 $\mu \mathrm{m}$ broad; valves are elongate, linear to lanceolate with rounded apices; striae is not clear.

Voucher no.: CUH/PLANK/DIA-34/1

\section{DISCUSSION}

Diatoms are widely used as environmental indicators in nano technology, oil exploration and forensic examinations (Dwivedi \& Misra, 2015). Recently the emphasis has been made on the ecological problems such as eutrophication, acidification and climate change. Freshwater diatom flora in ecologically sensitive regions of Indian continent has been discussed with very few reports Dwivedi \& Misra (2015) has reported 31 diatom species from the Himalayan region of which Encyonema subalpinum and Gomphonema towutense were found to be new to India. In our study we have also reported 31 species from $\mathrm{Cu}$ mining sites of Ghatshila of which 10 were newly recorded to the state. Pandey et al (2014) have studied 19 periphytic diatom taxa from the river Ganges polluted with $\mathrm{Cu}, \mathrm{Pb}$ and $\mathrm{Zn}$.

Four types of abundance categories have been proposed in the present study: (i) most dominant, (ii) dominant, (iii) less dominant and (iv) absent, on the basis of the contaminated sites. This pattern of study is consistent with Cattaneo et al. (2011). Diatom diversity was highest in all three sampling stations that were affected by $\mathrm{Cu}$ contamination. It has also been reported that the high level of $\mathrm{Cu}$, $\mathrm{Cd}$ and $\mathrm{Zn}$ can stimulate the growth of diatoms and increase their diversity (Sbihi et al., 2014). Interestingly, the present study supported the point of view of Sbihi et al. (2014). In station 1, 17 species were found to be dominant where $\mathrm{Cu}$ contamination was high. Among these, Halamphora coffeiformis was recorded as the most dominant species and not reported in station 2 and 3 . These tolerant taxa did not only survive under high $\mathrm{Cu}$ contamination but also showed their absolute abundance. Five species were less dominant and 9 were completely absent in station 1, which shows their low abundance in high $\mathrm{Cu}$ contamination $(400 \mu \mathrm{g} . \mathrm{L}$ $\left.{ }^{1}\right)$. In contrast to the remarkable $\mathrm{Cu}$ tolerance, only 9 species dominated in the medium $\mathrm{Cu}$ contaminated sites of station 2. Some of these species has confirmed their presence in the high $\mathrm{Cu}$ contaminating sites and some of them were completely absent in the area adjacent to HCL. It has been documented that some adnate species like Achnanthes minutissima and Fragilaria vaucheriae were found to be abundant in high Cu streams (Medley \& Clements, 1998). The growths of coastal and oceanic diatoms were highly affected by $\mathrm{Cu}$. Several coastal species like Chaetoceros decipiens, Thalassiosira weissflogii, Skeletolema costatum and many oceanic species like T. pseudonana, T. oceanica, S. menzellii were found to be rich in $\mathrm{Cu}$ infected areas (Annett et al., 2008). These results interpret that diatom tolerance to metals obviously has limits and their diversity fluctuates with the $\mathrm{Cu}$ level. Ecological characteristics such as optima and tolerance along with other environmental variables suggests diatom as ecological indicator (Dixit $\boldsymbol{e t}$ al., 1992). In station 3, only 5 species viz., Nitzschia acicularis, Amphora elliptica, Himantidium minus, Synedra ulna and S. ulna var. amphirhynchus showed their dominance over the less $\mathrm{Cu}$ contaminated area. These species were found as sensitive to high $\mathrm{Cu}$ (Table 2). Diatoms respond rapidly to environmental abnormalities and ecological fluctuations. Diatoms immigrate and replicate rapidly to any environmental changes. Changes in diatom assemblage in the present study sites correspond closely to shifts in other living communities such as picoplanktons, phytoplanktons, zooplanktons, fishes and hydrophytes (Dixit $\boldsymbol{e} t$ al., 1992).

Limnological parameter greatly influences the assemblage of diatoms an reduces water quality (Bigler $\boldsymbol{\&}$ Hall, 2002). It has been suggested that the diatoms respond well to hardness, alkalinity, $\mathrm{pH}$, salinity and nutrients (Greenaway et al., 2012). The appearance of low $\mathrm{pH}$ in water bodies indicates acidification. In our study we have observed the $\mathrm{pH}$ value in station 1 was 6.8 , which is slightly acidic suggesting the starting point of acidification due to pollution (Petrou et al., 2019). High acidification also diminishes silica production in diatoms (Petrou et al., 2019). Minimum value of calcium in station 1 suggests the low $\mathrm{pH}$. Total hardness and turbidity values in all stations suggest mineralization and eutrophic condition of the water bodies. Direct pouring of mining wastewater into the canals, outlets and in the River of Ghatshila results in significant changes to nitrate, phosphate, sulphate and silicate levels. High nitrate and sulphate values indicate the eutrophic condition of the water bodies of the sampling sites either due to anthropogenic activities or industrial pollution (Bella et al., 2007). Diatom communities play a significant role in maintaining the water quality in India. Some workers have concluded that diatoms can serve as indicator of organic and anthropogenic pollution (Choudhury \& Pal, 2010). The photosynthetic activity of the diatoms is directly associated with DO of the water. The productivity in relation to gross primary productivity (GPP) and net primary productivity (NPP) is directly correlated with DO. Rise in DO level in water results in high GPP, whereas rise in BOD is a measure of drop in NPP in the concerned water bodies. In our study DO value sharply increased in the order: station $1>$ station $2>$ station 3 , indicating the pollution level in the water Similarly BOD level decreased in the order: station $1<$ station $2<$ station 3 . As diatoms are primary producer they respond quickly to the ecological perturbations such as changing $\mathrm{n}$ physico-chemical parameters due to pollution (Adon, Quattara \& Gourene, 2012). High value of dissolved $\mathrm{CO}_{2}$ in station 1 adjacent to HCL indicates the competition of zooplanktons and other aquatic animals with the diatom communities for respiration. Poor water quality of station 1 due to contamination of $\mathrm{Cu}$ suggests diatom shift to the neighboring area of station 2 and 3. At some places diatoms can tolerate high metals whereas it drastically changes species composition in other places (Cattaneo et al., 2011) The conversion of oligotrophic to mesotrophic environment results in changing of species composition and species richness (Cumming $\boldsymbol{e t}$ al., 1995). In our study it was sharply observed that the dominant species in station 3 vanished completely in station 1 , which was highly polluted with $\mathrm{Cu}$ (Table 2). Similarly, species of highly $\mathrm{Cu}$ infected area were not observed in the less polluted area (station 3). Hence our study mainly focused on taxonomic implications of diatoms in $\mathrm{Cu}$ infected area with changing water quality.

\section{CONCLUSION}

Study of euplanktonic diatom communities in $\mathrm{Cu}$ mining sites is scarce. The present study was undertaken as first documentation of diatom communities in Jharkhand, India. The site is rich in $\mathrm{Cu}$ as it is situated adjacent to HCL on the bank of Subarnarekha River. A total number of 31 diatom species were documented from three sampling stations distinguished by high, medium and low $\mathrm{Cu}$ contamination. Maximum species dominated in station 1, which is closely associated to HCL. The taxonomic shifts were sharply noticed in three sampling sites. Oligotrophic to mesotrophic habitat suggests the eutrophication, acidification and mineralization of the water ecosystems. Physico-chemica parameters show the condition of the water bodies due to $\mathrm{Cu}$ contamination. From the study it can be concluded that the diatom assemblages of Ghatshila can serve as best bioindicator of $\mathrm{Cu}$.

Acknowledgments: Authors are thankful to the Department of Botany, Bangabasi Evening College and Phycology laboratory, University of Calcutta for instrumental facilities. Mrs. Mousumi Panda is thankfully acknowledged for English editing of this manuscript.

\section{REFERENCES}

Aboal, M., Álvarez Cobelas, M., Cambra, J. \& Ector, L. (2003). Floristic list of non-marine diatoms (Bacillariophyceae) of Iberian Peninsula, Balearic Islands and Canary Islands. Updated taxonomy and bibliography. Diatom Monographs, A. R. G. Gantner Verlag K.G., 4, 1-639.

Absil, M. C. P., Kroon, J. J. \& Wolterbeek, H. T. (1994). Availability of copper from phytoplankton and water for the bivalve Macoma balthica II. Uptake and elimination from $64 \mathrm{Cu}$ labelled diatoms and water. Marine Biology, 118, 129135. http://dx.doi.org/10.1007/BF00699227.

Adon, M. P., Quattara, A. \& Gourene, G. (2012). Phytoplankton composition of a shallow African tropical reservoir (ADZOPÉ, CÔTE D'IVOIRE). Journal of Microbiology, Biotechnology and Food Sciences, 1(5), 1189-1204.

American Public Health Administration (APHA). (1998). Standard methods for the examination of water and waste water. $\left(19^{\text {th }}\right.$ ed.), Washington, D. C.

Annett, A. L., Lapi, S., Ruth, T. J. \& Maldonado, M. T. (2008). The effects of Cu and $\mathrm{Fe}$ availability on the growth and $\mathrm{Cu}: \mathrm{C}$ ratios of marine diatoms. Limnology and Oceanography, 53(6), 2451-2461.

http://dx.doi.org/10.4319/lo.2008.53.6.2451.

Aponte, G. A., Maidana, N. I. \& Lange-Bertalot, H. (2005). On the taxonomic identity of Diadesmis confervaceoides Lange-Bert. et U. Rumrich (Bacillariophyceae). Cryptogamie Algologie, 26, 337-342.

Arguelles, E. D. L. R. (2019). Morphotaxonomic Study of Algal Epiphytes from Ipomea aquatica Forssk. (Convolvulaceae) Found in Laguna de Bay (Philippines). Pertanika Journal of Tropical Agricultural Science, 42(2), 817 832.

Arguelles, E. D. L. R. (2020). Species Composition of Algal Epiphyton of Pink Lotus (Nymphaea pubescens Willd) Found in Laguna de Bay (Philippines) Walailak Journal of Science and Technology, 17(3): 237-256. 
Bella, V. D., Puccinelli, C., Marcheggiani, S. \& Mancini, L. (2007). Benthic diatom communities and their relationship to water chemistry in wetlands of Central Italy. Annales de Limnologie- International Journal of Limnology, 43(2), 89-99. http://dx.doi.org/10.1051/limn/2007021.

Bhakta, S., Das, S. K., Nayak, M., Jena, J., Panda, P. K. \& Sukla, L. B. (2011). Phyco-Diversity Assesssment of Bahuda River Mouth Areas of East Coast of Odisha, India. Recent Research in Science and Technology, 2(4), 80-89.

Bhakta, S., Das, S. K. \& Adhikary, S. P. (2016). Algal diversity in hot springs of Odisha. Nelumbo, 58, 157-173.

http://dx.doi.org/10.20324/nelumbo/v58/2016/105914

Bigler, C., \& Hall, R. I. (2002). Diatoms as indicators of climatic and limnological change in Swedish Lapland: a 100-lake calibration set and its validation for paleoecological reconstructions. Journal of Paleolimnology, 27, 97-115. http://dx.doi.org/10.1023/A:1013562325326.

Bose, R., Bar, R. \& Pal, R. (2017). Floristic Assortment of Planktonic and Epipsammic Diatoms from Eastern India with New Reports. Journal of Algal Biomass Utilization, 8(4), 51-68.

Cattaneo, A., Couillard, Y., Wunsam, S. \& Fortin, C. (2011). Littoral diatoms as indicators of recent water and sediment contamination by metals in lakes. Journa of Environmental Monitoring, 13, 572-582.

http://dx.doi.org/10.1039/C0EM00328J.

Cheng, Z. \& Gao, Y. (2012). Flora algarum marinarum sinicarum. Tomus V. Bacillariophyta No. I. Centricae. pp. i-viii, i-xxvi, 1-137, 35 pls. Beijing: Science Press.

Chmielewska, E. \& Medved, J. (2001). Bioaccumulation of Heavy metals by green algae Cladophora glomerata in a refinery sewage lagoon. Croatica Chemica Acta, 74(1), 135-145.

Cholnoky, B. J. (1956). Neue und seltene Diatomeen aus Afrika. II. Diatomeen aus dem Tugela-Gebiete in Natal. Österreichische Botanische Zeitschrift, 103 , 53-97.

Choudhury, A. K. \& Pal, R. (2010). Phytoplankton and nutrient dynamics of shallow coastal stations at Bay of Bengal, Eastern Indian coast. Aquatic Ecology, 44, 55-71. http://dx.doi.org/10.1007/s10452-009-9252-9.

Cleve, P. T. (1895). Synopsis of the Naviculoid Diatoms, Part II. Kongliga Svenska-Vetenskaps Akademiens Handlingar, 27(3), 1-219.

Cocquyt, C., Kusber, W. -H. \& Jahn, R. (2018). Epithemia hirudiniformis and related taxa within the subgenus Rhopalodiella subg. nov. in comparison to Epithemia subg. Rhpalodia stat nov. (Bacillariophyceae) from East Africa. Cryptogamie Algologie, 39(1), http://dx.doi.org/10.7872/crya/v39.iss1.2018.35.

Cumming, B. F., Wilson, S. E., Hall, R. I. \& Smol, J. P. (1995). Diatoms from British Columbia (Canada) lakes and their relationship to salinity, nutrients and other limnological vari- ables. Bibliotheca Diatomologica, Volume 31. J. Cramer, Berlin, Stuttgart

Das, S. K. \& Adhikary, S. P. (2012). Freshwater algae of Cherrapunjee and Mawsynram, the wettest places on Earth. Phykos, 44(2), 29-43.

Dixit, S. S., Smol, J. P., Kingston, J. C. \& Charles, D. F. (1992). Diatoms: powerful indicators of environmental change. Environmental Science \& Technology, 26(1), 22-33. http://dx.doi.org/10.1021/es00025a002.

Dwivedi, R. K. \& Misra, P. K. (2015). Fresh Water Diatoms from Himalayan State, Himachal Pradesh, India. Phykos, 45(1), 30-39.

Eberle, M. E. (2008). Recent diatoms reported from the central United States: register of taxa and synonyms. Hays, Kansas: Department of Biological Sciences, Fort Hays State University. Latest electronic version: 5 December 2008.

Gandhi H. P. 1959. Fresh water diatoms from Sagar in Mysore state. Journal of the Indian Botanical Society, 38, 305-331.

Gandhi H. P. 1967. Notes on the Diatomaceae from Ahmedabad and its environs VI. On some diatoms from fountain reservoir of Seth Sarabhai's garden Hydrobiologia, 30(2), 248-272. http://dx.doi.org/10.1007/BF00034596.

Greenaway, C. M., Paterson, A. M., Keller, (Bill) W. \& Smol, J. P. (2012) Dramatic diatom species assemblage responses in lakes recovering from acidification and metal contamination near Wawa, Ontario, Canada: a paleolimnological perspective. Canadian Journal of Fisheries and Aquatic Sciences, 69(4), 656-669. http://dx.doi.org/10.1139/f2012-003.

Guiry, M. D. in Guiry, M. D. \& Guiry, G. M. (2021). World-wide electronic publication, National University of Ireland, Galway. AlgaeBase, searched on 04 February 2021, http://www.algaebase.org.

Hendey, N. I. (1974). A revised check-list of the British marine diatoms. Journal of the Marine Biological Association of the United Kingdom, 54, 277-300. http://dx.doi.org/10.1017/S0025315400058549.

Hofmann, G., Werum, M. \& Lange-Bertalot, H. (2013). Diatomeen im Süßwasser-Benthos von Mitteleuropa. Bestimmungsflora Kieselalgen für die ökologische Praxis. Über 700 der häufigsten Arten und ihre Ökologie. pp. [1] 908, 133 pls. Königstein: Koeltz Scientific Books.

https://www.google.com/maps/search/ghatshila,+hindustan+copper+limited/@22 $.5911511,86.4454325,4927 \mathrm{~m} /$ data $=! 3 \mathrm{~m} 1 ! 1 \mathrm{e} 3$

Hustedt, F. (1930). Bacillariophyta (Diatomeae) Zweite Auflage. In: Die Süsswasser-Flora Mitteleuropas. Heft 10. (Pascher, A. Eds), pp. i-vii, 1-466. Jena: Verlag von Gustav Fischer.
Hu, H. \& Wei, Y. (2006). The freshwater algae of China. Systematics, taxonomy and ecology. pp. i-iv, i-xv, 1-1023, 4 pls, 16 figs,China: www.sciencep.com.

Jahn, R. \& Kusber, W. H. (2004). Algae of the Ehrenberg collection - 1 Typification of 32 names of diatom taxa described by C.G. Ehrenberg. Willdenowia, 34(2), 577-595. http://dx.doi.org/10.3372/wi.34.34219. John, J. (2018). Diatoms from Tasmania: taxonomy and biogeography. The diatom flora of Australia Volume 2. pp. 1-656, 351 figs. Schmitten Oberreifenberg: Koeltz Botanical Books.

Kociolek, J. P., Balasubramanian, K., Blanco, S., Coste, M., Ector, L., Liu, Y., Kulikovskiy, M., Lundholm, N., Ludwig, T., Potapova, M., Rimet, F., Sabbe, K Sala, S., Sar, E., Taylor, J., Van de Vijver, B., Wetzel, C. E., Williams, D. M. Witkowski, A. \& Witkowski, J. (2018). DiatomBase. Amphora ovum Cleve, 1895 Accessed

http://www.diatombase.org/aphia.php?p=taxdetails\&id=646200 on 2020-03-24

Krammer, K. \& Lange-Bertalot. (2004). Süßwasserflora von Mitteleuropa. Band 2. Bacillariophyceae. Teil 4: Acnanthaceae. Kritische Ergänzungen zu Achnanthes s.l., Navicula s. str., Gomphonema. pp. [i-vi], 1-468, 93 pls. Heidelberg \& Berlin: Spektrum Akademischer Verlag.

Kulikovskiy, M., Glushchenko, A. M., Kuznetsova, I. V. \& Genkal, S. I (2016). Identification book of diatoms from Russia. pp. 1-804, 165 pls. Yaroslavl: Filigran.

Lange-Bertalot, H., Hofmann, G., Werum, M. \& Cantonati, M (2017). Freshwater benthic diatoms of Central Europe: over 800 common species used in ecological assessments. English edition with updated taxonomy and added species (Cantonati, M. et al. eds). pp. 1-942, 135 pls. SchmittenOberreifenberg: Koeltz Botanical Books.

Lee, S. S., Gaiser, E. E., Van de Vijver, B., Edlund, M. B. \& Spaulding, S. A (2014). Morphology and typification of Mastogloia smithii and M. lacustris, with descriptions of two new species from the Florida Everglades and the Caribbean region. Diatom Research, 29(4), http://dx.doi.org/10.1080/0269249X.2014.889038.

Levkov, Z. (2009). Amphora sensu lato. In: Diatoms of Europe: Diatoms of the European Inland Waters and Comparable Habitats. (Lange-Bertalot, H. Eds) Vol. 5, pp. 5-916. Ruggell: A. R. G. Gantner Verlag K. G.

Li, J. \& Qi, Y. (2018). Flora algarum sinicarum aquae dulcis Tomus XXIII Bacillariophyta Naviculaceae (III). In (Li, J. Y. \& Qi, Y. Z.). pp. [1-8], i-xxiii, 1 214, pls I-XLVIII. Beijing: Science Press.

Medley, C. N. \& Clements, W. H. (1998). Responses of diatom communities to heavy metals in streams: the influence of longitudinal variations. Ecological Applications, 8(3), 631-644. http://dx.doi.org/10.2307/2641255.

Miscoe, L. H., Johansen, J. R., Kociolek, J. P. \& Lowe, R. L. (2016). The diatom flora and cyanobacteria from caves on Kauai, Hawaii. I. Investigation of the cave diatom flora of Kanuai, Hawaii: an emphasis on taxonomy and distribution. Bibliotheca Phycologica, 123, 3-74.

Nor, Y. M. (1987). Ecotoxicity of copper to aquatic biota: a review. Environmental Research, 43(1), 274-282. http://dx.doi.org/10.1016/S0013 9351(87)80078-6.

Pandey, L. K., Kumar, D., Yadav, A., Rai, J. \& Gaur, J. P. (2014). Morphological abnormalities in periphytic diatoms as a tool for biomonitoring of heavy metal pollution in a river. Ecological Indicators, 36, 272-279. http://dx.doi.org/10.1016/j.ecolind.2013.08.002.

Patrick, R. M. \& Reimer, C. W. (1966). The diatoms of the United States exclusive of Alaska and Hawaii. Volume 1: Fragilariaceae, Eunotiaceae, Achnanthaceae, Naviculaceae. Monographs of the Academy of Natural Sciences of Philadelphia, 13, 1-688.

Petrou, K., Baker, K. G., Nielsen, D. A., Hancock, A. M., Schulz, K. G. \& Davidson, A. T. (2019). Acidification diminishes diatom silica production in the Southern Ocean. Nature Climate Change, 9, 781-786. http://dx.doi.org/10.1038/s41558-019-0557-y.

Proschkina-Lavrenko, A. I. (1950). Diatomovyi Analiz, Kniga 3. Opredelitel iskopaemykh i sovremennykh diatomik vodoroslei Poriadok Pennales. Botanicheskii Institut im V.L. Komarova Akademii Nauk S.S.S.R Gosudarstvennoe Izdatelystvo Geologicheskoi Literatury, 3(1), 1-398.

Ruck, E. C., Nakov, T., Alverson, A. J. \& Theriot, E. C. (2016). Nomenclatural transfers associated with the phylogenetic reclassification of the Surirellales and Rhopalodiales. Notulae Algarum, 10, 1-4.

Satpati, G. G., Barman, N. \& Pal, R. (2012). Morphotaxonomic account of some common seaweed from Indian Sundarbans mangrove forest and inner island area. Journal of Algal Biomass Utilization, 3(4), 45-51.

Satpati, G. G., Barman, N. \& Pal, R. (2013). A study on green algal flora of Indian Sundarbans mangrove forest with special reference to morphotaxonomy. Journal of Algal Biomass Utilization, 4(1), 26-41.

Satpati, G. G \& Pal, R. (2016). New and rare records of filamentous green algae from Indian Sundarbans Biosphere Reserve. Journal of Algal Biomass Utilization, 7(2), 159-175.

Satpati G. G. \& Pal, R. (2017). Taxonomic diversity and SEM study of Euglenoids from brackish water ecosystems of Indian Sundarbans Biosphere Reserve. Phykos, 47(1), 105-122.

Satpati, G. G., Gorain, P. C., Sengupta, S. \& Pal, R. (2017). Cocconei gracilariensis sp. nov. (Cocconeidaceae, Bacillariophyta) from India: a new 
brackish water diatom, epiphytic on invasive red seaweed Gracilaria sp. NeBIO, 8(2), 88-93.

Satpati, G. G. (2021). Solid waste management by algae: Current applications and future perspectives. Pollution Research, 40(1): 260-265. In press.

Sbihi, K., Cherifi, O., Bertand, M. \& El Gharmali, A. (2014). Biosorption of metals $(\mathrm{Cd}, \mathrm{Cu}$ and $\mathrm{Zn})$ by the freshwater diatom Planothidium lanceolatum: a laboratory study. Diatom Research, 1-9. http://dx.doi.org/10.1080/0269249X.2013.872193.

Slate, J. \& Stevenson, R. J. (2007). The diatom flora of phosphorus-enriched and unenriched sites in a Everglades marsh. Diatom Research,22(2), 355-386. http://dx.doi.org/10.1080/0269249X.2007.9705721.

Stepanek, J. G. \& Kociolek, J. P. (2018). Amphora and Halamphora from coastal waters and inland waters of the United States and Japan. Bibliotheca Diatomologica, 66, 1-260.

Van Heurck, H. (1881). Synopsis des Diatomées de Belgique Atlas. pls XXXILXXVII. Anvers: Ducaju et Cie.

Venkataraman, G. (1939). A systematic account of some south Indian diatoms.

Proceedings of the Indian Academy of Sciences, Section B, 10, 293-368.

Wang, P., Park, B. S., Kim, J. H., Kim, J. -H., Lee, H. -O. \& Han, M. -S. (2014)

Phylogenetic position of eight Amphora sensu lato (Bacillariophyceae) species and comparative analysis of morphological characteristics. Algae, 29(2), 57-73. http://dx.doi.org/10.4490/algae.2014.29.2.057

Winkler, L. W. (1888). The determination of dissolved oxygen in water. Berichte der Deutschen Chemischen Gesellschaft, 21, 2843.

Witkowski, A., Lange-Bertalot, H. \& Metzeltin, D. (2000). Diatom flora of marine coasts I. Iconographia Diatomologica, 7, 1-925.

Zeraatkar, A. K., Ahmadzadeh, H., Talebi, A. F., Moheimani, N. R. \& McHenry,

M. P. (2016). Potential use of algae for heavy metal bioremediation, a critical review. Journal of Environmental Management, 181, 817-831. http://dx.doi.org/10.1016/j.jenvman.2016.06.059. 